\title{
Large soutien pour la campagne smarter medicine
}

\section{Organisation responsable "smarter medicine»}

\section{Une nouvelle association de soutien a été créée à la mi-juin afin de donner une} nouvelle impulsion en Suisse à la campagne smarter medicine lancée il y a trois ans. Les orientations de la campagne sont soutenues par des organisations médicales spécialisées et professionnelles, mais aussi par des associations qui défendent les intérêts des patients et des consommateurs. Ensemble, elles souhaitent sensibiliser le public au fait qu'en médecine et pour certains traitements, moins peut parfois signifier plus de qualité de vie pour les personnes concernées.

Des voix se sont fait entendre il y a quelques années aux Etats-Unis afin de mettre en garde contre les conséquences de l'excès de soins médicaux («overuse»). En 2011, des médecins ont lancé l'initiative "Choosing Wisely". Celle-ci a pour but non seulement de déboucher sur des «décisions judicieuses», mais aussi d'encourager une franche discussion entre le corps médical, les patients et le public.

«Choosing Wisely" s'articule autour de ce qu'on appelle des «listes Top 5» dans chaque discipline clinique. Ces listes Top 5 comprennent chacune cinq mesures médicales qui sont généralement inutiles. En d'autres termes, les médecins et les patients devraient discuter afin de déterminer s'il ne vaut pas mieux renoncer à un traitement, parce que les risques afférents sont potentiellement plus élevés que son utilité.

L'Académie Suisse des Sciences Médicales (ASSM) a diffusé l'initiative "Choosing Wisely", dans le cadre de sa feuille de route "Médecine durable». Cette question a également suscité un vif intérêt auprès de la Société Suisse de Médecine Interne Générale (SSMIG). Une commission a par la suite élaboré sa propre liste Top 5 pour le domaine ambulatoire en Suisse et l'a présentée au public en 2014, sous le nom de smarter medicine. Elle a été suivie deux ans plus tard par la publication d'une liste Top 5 pour le domaine hospitalier.

Malgré tout, la campagne smarter medicine n'a pas vraiment réussi à prendre pied en Suisse, contrairement à d'autres pays, et n'a guère été soutenue par d'autres sociétés de discipline médicale. C'est la raison pour laquelle la SSMIG et l'ASSM ont pris l'initiative de créer un mouvement de soutien plus vaste de la campagne en Suisse. Les patients et les consommateurs ainsi que les autres professions de santé devraient notamment être impliqués.
L'association de soutien smarter medicine sera à présent créée en juin 2017. Outre la SSMIG et l'ASSM, elle compte également parmi ses membres la Fédération Suisse des Associations professionnelles du domaine de la Santé (FSAS), la Fédération Suisse des Patients (FSP), ainsi que les organisations de consommateurs Fondation pour la protection des consommateurs (FPC), Fédération Romande des Consommateurs (FRC) et Associazione consumatrici e consumatori della Svizzera italiana (acsi).

L'association de soutien poursuit les objectifs suivants:

- élaboration et publication de listes Top 5 supplémentaires par les sociétés de discipline médicale, etc.;

- renforcer le caractère contraignant des recommandations;

- impliquer activement d'autres professions de santé (approche interprofessionnelle);

- sensibiliser les patients et les clients à la question;

- initier un débat public sur la qualité des soins (excès ou déficit de soins);

- instaurer et ancrer le thème de la qualité des traitements comme composante de la formation postgraduée et continue;

- obtenir le soutien de la campagne par la politique et les autorités.

Il est très important pour les membres fondateurs de l'association de soutien que la campagne smarter medicine ne soit pas accaparée par l'économie de la santé. Il s'agit avant tout d'améliorer la qualité des soins, conformément à la devise qu'«en médecine, moins peut aussi être plus».

Vous trouverez de plus amples informations concernant la cam pagne et l'association de soutien sur www.smartermedicine.ch. 